\title{
АНАЛИЗ ЗАБОЛЕВАЕМОСТИ МНОЖЕСТВЕННОЙ МИЕЛОМОЙ В РЕСПУБЛИКЕ БЕЛАРУСЬ
}

\author{
(C) Чешик A.A.
}

\author{
Государственный регистр лиц, подвергшихся воздействию радиации вследствие катастрофы \\ на Чернобыльской АЭС Республиканского научно-практического центра радиационной медицины \\ и экологии человека, Гомель, Республика Беларусь
}

E-mail: acheshik@tut.by

Целью работы явилась оценка заболеваемости множественной миеломой (МКБ10: С90) в Республике Беларусь в 1991-2014 гг. В работе были проанализированы данные Белорусского республиканского канцер-регистра за период с 1991-го по 2014 г. Рассчитаны грубые интенсивные (CR), повозрастные (AsR) и стандартизованные по возрасту (ASR) показатели заболеваемости множественной миеломой (на 100000 населения). Анализ динамик показателей проводился путем сравнения темпов среднегодового прироста (APC). При анализе ASR было показано, что заболеваемость у мужчин выше, чем у женщин, в отличии от CR. Между тем заболеваемость городского населения, как мужчин так и женщин, оказалась выше, чем у сельского населения, у обоих показателях. Анализ AsR показал, что риск заболевания в первые 30 лет жизни минимален. После 35 лет заболеваемость резко увеличивается с возрастом, достигая максимума в возрастной группе 70-74 года. После 75 лет заболеваемость множественной миеломы начинает снижаться.

Ключевые слова: множественная миелома, заболеваемость, стандартизованный показатель, грубый интенсивный показатель.

\section{MULTIPLE MYELOMA INCIDENCE RATES IN THE REPUBLIC OF BELARUS \\ Cheshik A.A.}

State Register of Persons Exposed to Radiation as a Result of the Chernobyl Disaster of Republican Scientific and Practical Center for Radiation Medicine and Human Ecology, Gomel, Republic of Belarus

Multiple myeloma is one of the most expected malignant neoplasms, which could be associated with radiation impact. Thus the aim of this study was to estimate multiple myeloma rates in Belarus within 1991-2014. Data of Belorussian Cancer Registry were analyzed by calculating crude rates (CR), age-specific rates (AsR) and age standardized rates (ASR). Trend analysis was realized by investigating average annual percentage change (APC). In analyzing AsR the risk of the disease in the first 30 years of life is minimum. After 35 years the risk is dramatically growing. After 75 years the risk of multiple myeloma starts decreasing. Dynamic parameters have grown in both crude and standardized incidence rates (APC is about $3.5 \%$ ). CR is higher in males than females as opposed to ASR. CR and ASR are higher for urban rather than rural residents. The data obtained can be used in further epidemiological studies of multiple myeloma risk in the groups of high radiation exposure.

Keywords: multiple myeloma, standardized incidence rates, crude incidence rates.

Известно, что наиболее ожидаемым эффектом ионизирующей радиации является индукция злокачественных новообразований лимфоидной, кроветворной и родственных им тканей [1]. По данным Международного агентства по изучению рака МАИР [4], заболеваемость множественной миеломой (ММ) в мировой популяции мужчин в 2012 году составила $1,7 \% 0000$, у женщин $-1,2 \%$. В то же время отмечено, что показатель заболеваемости у мужчин в развивающихся странах $(1,0 \% 0000)$ в три раза ниже, чем у мужского населения развитых стран $(3,3 \% 000)$. Среди женского населения различия в уровнях заболеваемости в развитых $(2,2 \%$ и 2000$)$ и развивающихся $(0,7 \% 0000)$ странах находятся примерно на том же уровне, что и у мужчин.

Необходимо отметить высокую (по отношению к заболеваемости) смертность от ММ в развивающихся странах $(0,8$ у мужчин и 0,7 у женщин). Соотношение же величины показателя смертности к величине показателя заболеваемос- ти у мужчин и женщин, проживающих в развитых странах ниже - 0,5.

В то же время проведенный нами анализ литературных источников показал небольшое количество публикаций, характеризующих тенденции заболеваемости ММ в Республике Беларусь в послеаварийный период. Знание особенностей заболеваемости ММ в Беларуси важно для решения задач по оценке радиационно обусловленных рисков развития этого заболевания у пострадавшего населения. В связи с этим целью данной работы было проведение анализа заболеваемости ММ в Республике Беларусь за период с 1991-го по 2014 г.

\section{МАТЕРИАЛЫ И МЕТОДЫ ИССЛЕДОВАНИЯ}

В работе были проанализированы данные Белорусского республиканского канцер-регистра 


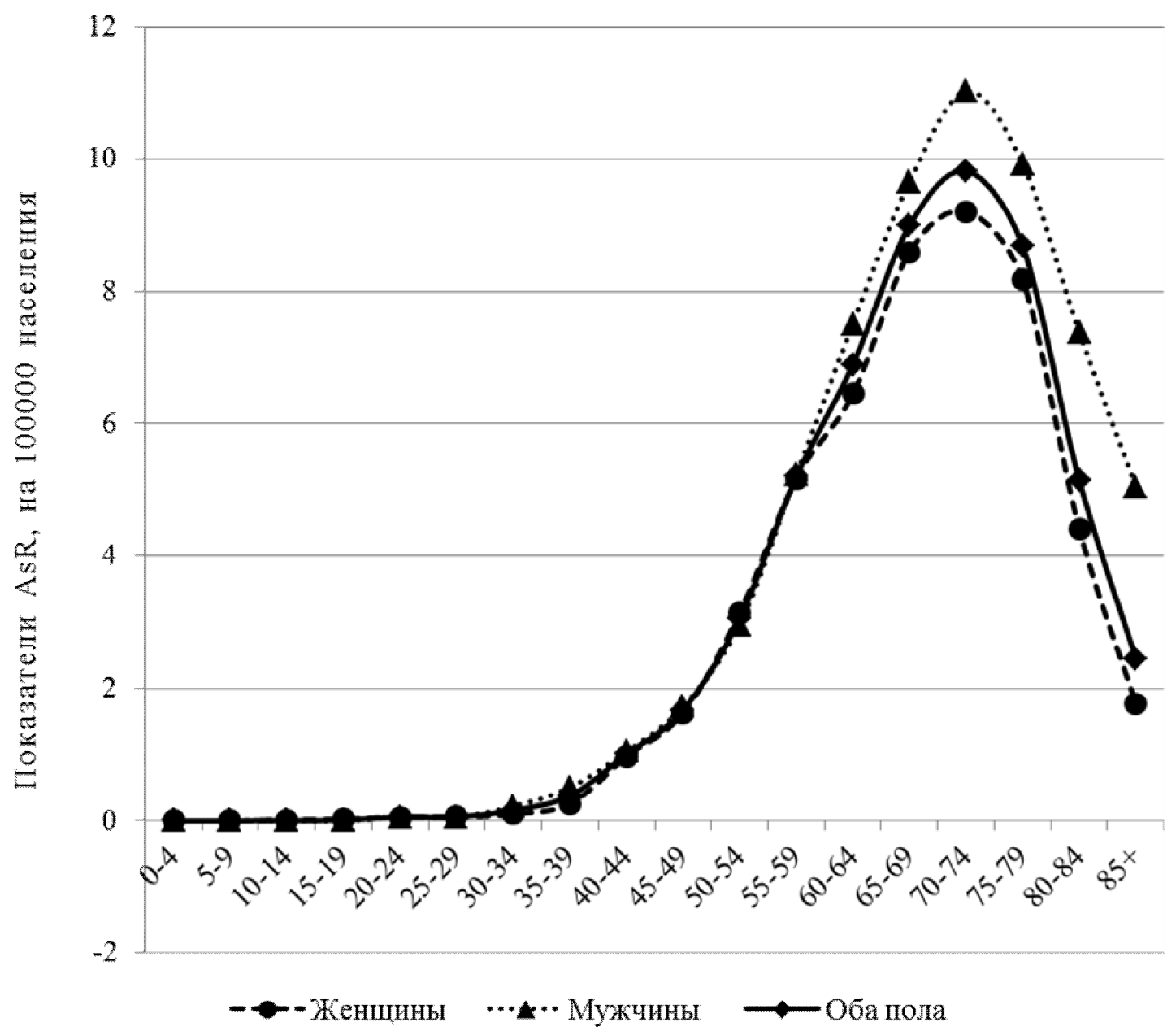

Рис. 1. Повозрастные показатели заболеваемости (AsR 95\% ДИ) MM в Республики Беларусь за период с 1991 по 2014 гг.

за период с 1991-го по 2014 г. В данной работе устанавливались особенности заболеваемости MM (С90). В работе была использована Международная классификация болезней 10 пересмотра. Были рассчитаны грубые интенсивные (CR), повозрастные (AsR) и стандартизованные по возрасту (ASR, World стандарт) показатели заболеваемости лейкозами (на 100000 населения). Статистическая обработка материала проводилась согласно стандартным методам, принятым в эпидемиологии злокачественных новообразований: сравнение показателей заболеваемости, проводилось с помощью Z-критерия, стандартной ошибки среднего (SE) и сравнения 95\% доверительных интервалов (95\% ДИ) [2, 3]. Анализ динамик показателей заболеваемости проводился путем сравнения темпов среднегодового прироста (APC).

\section{РЕЗУЛЬТАТЫ ИССЛЕДОВАНИЯ И ИХ ОБСУЖДЕНИЕ}

В структуре заболеваемости населения Беларуси злокачественными опухолями $\mathrm{MM}$
(С90) на протяжении 1991-2014 годов составляли порядка 0,3-0,4\%, как у мужчин, так и у женщин.

За период с 1991-го по 2014 год в Республике Беларусь зарегистрировано 5173 случая ММ. Соотношение числа заболевших мужчин и женщин за это время составило 0,73/1.

На рисунке 1 приведены повозрастные показатели заболеваемости ММ мужчин и женщин Республики Беларусь за период с 1991-го по 2014 г. Как видно из рисунка, показатели заболеваемости ММ выше у мужчин, чем у женщин, статистически значимо в возрастных группах 35-39 лет $(\mathrm{p}<0,05), 60-64$ года $(\mathrm{p}<0,05)$ и во всех возрастных группах старше 70 лет $(\mathrm{p}<0,05)$. Анализ повозрастных показателей заболеваемости показывает, что в отличие от многих других злокачественных новообразований риск заболевания в первые 30 лет жизни минимален $\quad(0,1 \pm 0,04 \%$ 0000). После 35 лет заболеваемость резко увеличивается с возрастом, достигая максимума в возрастной группе 70-74 года у мужчин $(11 \pm 1,19 \%$ о0о0) и у женщин $(9,2 \pm 0,78 \%$ о0о0). После 75 лет заболеваемость ММ начинает снижаться. 
Число случаев ММ в Беларуси с 1991-го по 2014 г. и среднегодовой темп прироста

\begin{tabular}{|c|c|c|c|c|c|c|c|}
\hline \multirow[b]{2}{*}{ Пол } & \multicolumn{6}{|c|}{ Годы } & \multirow[b]{2}{*}{$\begin{array}{c}\text { АРС (95\% ДИ) } \\
\%\end{array}$} \\
\hline & $\begin{array}{l}1991- \\
1994\end{array}$ & $\begin{array}{l}1995- \\
1999\end{array}$ & $\begin{array}{l}2000- \\
2004\end{array}$ & $\begin{array}{l}2005- \\
2009\end{array}$ & $\begin{array}{l}2010- \\
2014\end{array}$ & $\begin{array}{l}1991- \\
2014\end{array}$ & \\
\hline Мужчины & 245 & 377 & 441 & 528 & 600 & 2191 & $3,4(2,7-3,99)^{*}$ \\
\hline Женщины & 305 & 509 & 608 & 731 & 829 & 2982 & $3,6(2,9-4,31)^{*}$ \\
\hline Оба пола & 550 & 886 & 1049 & 1259 & 1429 & 5173 & $3,5(2,9-4,11)^{*}$ \\
\hline
\end{tabular}

Таблица 2 Динамики грубых интенсивных и стандартизованных показателей заболеваемости ММ мужчин и женщин, городских и сельских жителей, темпы среднегодового прироста

\begin{tabular}{|c|c|c|c|c|c|c|c|c|c|}
\hline \multirow{3}{*}{ 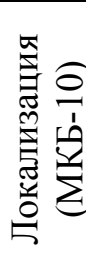 } & \multirow{3}{*}{$\begin{array}{c}\text { Пол/ } \\
\text { место } \\
\text { жительства }\end{array}$} & \multirow{2}{*}{\multicolumn{4}{|c|}{$\begin{array}{c}\text { Грубый интенсивный показатель } \\
\text { заболеваемости } \\
\text { на } 100000 \text { населения } \pm \mathrm{Z} \times \mathrm{Se}\end{array}$}} & \multicolumn{4}{|c|}{$\begin{array}{c}\text { Стандартизованный показатель } \\
\text { заболеваемости } \\
\text { на } 100000 \text { населения } \pm \mathrm{z} \times \mathrm{Se}\end{array}$} \\
\hline & & & & & & \multicolumn{4}{|c|}{ Годы } \\
\hline & & 1991 & 2001 & 2014 & $\begin{array}{c}\text { АРC } \\
(95 \% Д И)\end{array}$ & 1991 & 2001 & 2014 & $\begin{array}{c}\text { APC } \\
(95 \% Д И)\end{array}$ \\
\hline \multirow{6}{*}{ 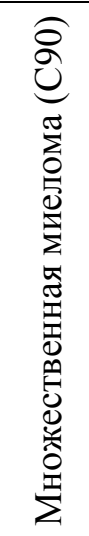 } & Женщины/все & $\begin{array}{c}1,5 \\
\pm 0,33\end{array}$ & $\begin{array}{c}2,5 \\
\pm 0,43\end{array}$ & $\begin{array}{c}3,3 \\
\pm 0,5\end{array}$ & $\begin{array}{c}3,9 \\
(3,24-4,65)^{*}\end{array}$ & $\begin{array}{c}1 \\
\pm 0,15\end{array}$ & $\begin{array}{c}1,4 \\
\pm 0,38\end{array}$ & $\begin{array}{c}1,7 \\
\pm 0,28\end{array}$ & $\begin{array}{c}2,8 \\
(2,09-3,55)^{*}\end{array}$ \\
\hline & $\begin{array}{l}\text { Женщины/ } \\
\text { город }\end{array}$ & $\begin{aligned} & 1,4 \\
& \pm 0,39 \\
&\end{aligned}$ & $\begin{array}{c}2 \\
\pm 0,45 \\
\end{array}$ & $\begin{array}{c}3,4 \\
\pm 0,58 \\
\end{array}$ & $\begin{array}{c}4,2 \\
(3,39-4,93)^{*}\end{array}$ & $\begin{array}{c}1,2 \\
\pm 0,21 \\
\end{array}$ & $\begin{array}{c}1,3 \\
\pm 0,83 \\
\end{array}$ & $\begin{array}{r}1,9 \\
\pm 0,42 \\
\end{array}$ & $\begin{array}{c}2,3 \\
(1,58-3,01)^{*}\end{array}$ \\
\hline & Женщины/село & $\begin{array}{c}1,7 \\
\pm 0,59 \\
\end{array}$ & $\begin{array}{c}3,8 \\
\pm 0,96 \\
\end{array}$ & $\begin{array}{c}3,1 \\
\pm 1,02 \\
\end{array}$ & $\begin{array}{c}3,4 \\
(2,17-4,67)^{*}\end{array}$ & $\begin{array}{c}0,8 \\
\pm 0,23 \\
\end{array}$ & $\begin{aligned} & 1,6 \\
& \pm 0,32 \\
&\end{aligned}$ & $\begin{array}{c}1,2 \\
\pm 0,26 \\
\end{array}$ & $\begin{array}{c}3,1 \\
(1,75-4,48)^{*}\end{array}$ \\
\hline & Мужчины/все & $\begin{array}{c}1,3 \\
\pm 0,32\end{array}$ & $\begin{array}{c}1,8 \\
\pm 0,38\end{array}$ & $\begin{array}{c}3 \\
\pm 0,52\end{array}$ & $\begin{array}{c}3,8 \\
(3,19-4,45)^{*}\end{array}$ & $\begin{array}{c}1,2 \\
\pm 0,17\end{array}$ & $\begin{array}{c}1,4 \\
\pm 0,18\end{array}$ & $\begin{array}{c}2,2 \\
\pm 0,2\end{array}$ & $\begin{array}{c}3 \\
(2,3-3,68)^{*}\end{array}$ \\
\hline & $\begin{array}{l}\text { Мужчины/ } \\
\text { город }\end{array}$ & $\begin{array}{c}1,3 \\
\pm 0,4\end{array}$ & $\begin{array}{c}1,8 \\
\pm 0,46\end{array}$ & $\begin{array}{c}3,2 \\
\pm 0,6\end{array}$ & $\begin{array}{c}4,1 \\
(3,3-4,94)^{*}\end{array}$ & $\begin{array}{c}1,5 \\
\pm 0,24\end{array}$ & $\begin{array}{c}1,6 \\
\pm 0,24\end{array}$ & $\begin{array}{c}2,5 \\
\pm 0,25\end{array}$ & $\begin{array}{c}2,5 \\
(1,61-3,42)^{*}\end{array}$ \\
\hline & Мужчины/село & $\begin{array}{c}1,2 \\
\pm 0,55\end{array}$ & $\begin{array}{c}1,7 \\
\pm 0,7\end{array}$ & $\begin{array}{c}2,7 \\
\pm 0,99\end{array}$ & $\begin{array}{c}3,1 \\
(2,08-4,06)^{*}\end{array}$ & $\begin{array}{c}1 \\
\pm 0,34\end{array}$ & $\begin{array}{c}0,9 \\
\pm 0,21\end{array}$ & $\begin{array}{c}1,6 \\
\pm 0,37\end{array}$ & $\begin{array}{c}2,8 \\
(1,59-4,03)^{*}\end{array}$ \\
\hline
\end{tabular}

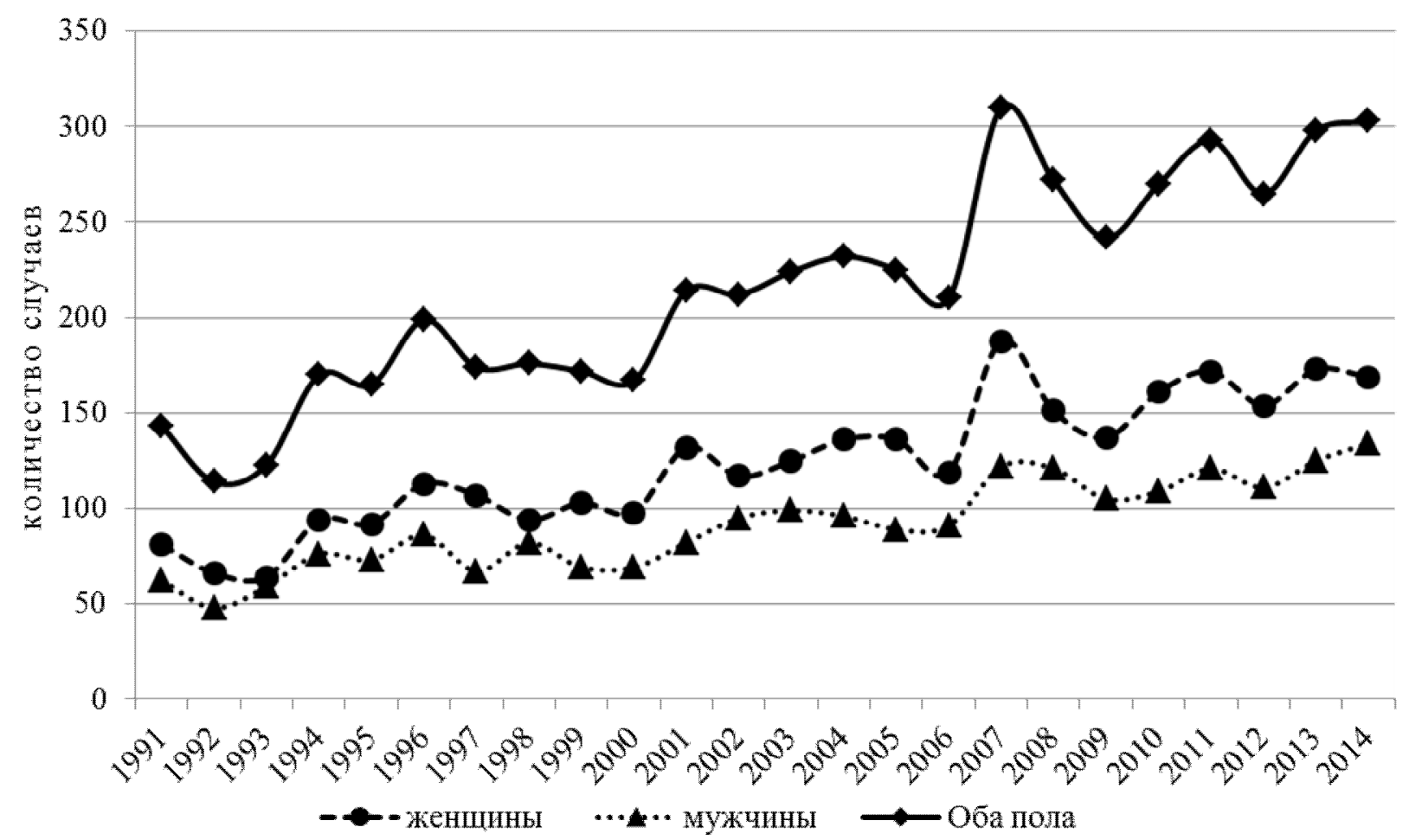

Рис. 2. Динамика ежегодно выявляемых случаев ММ в РБ. 
Проведенный анализ заболеваемости ММ в динамике показал, что в течение всего периода наблюдения с 1991-го по 2014 г. наблюдался рост числа ежегодно заболевших ММ на территории Республики Беларусь. Так, число ежегодно регистрируемых новых случаев заболевания в 1991 году составляло 143 (62 у мужчин и 81 у женщин), а в 2014-м - 303 случая (134 у мужчин и 169 у женщин) (среднегодовой темп прироста составил $($ АРС $=3,5 \quad(2,9-4,11) \% \quad(\mathrm{p}<0,05))$. Таблица 1, рисунок 2)).

В таблице 2 приведены динамики грубых интенсивных и стандартизованных по возрасту показателей заболеваемости ММ мужчин и женщин, городских и сельских жителей.

Динамика грубого интенсивного показателя заболеваемости ММ имеет статистически значимые положительные среднегодовые темпы прироста более $3 \%$ как у мужчин, так и у женщин, жителей города и села. Как видно из таблицы, грубый интенсивный показатель заболеваемости у мужчин вырос с $1,3 \pm 0,32 \%$ в 1991 г. до $3 \pm 0,52 \%$ в 2014 г. $($ APC $=3,8 \quad(3,19-4,45) \%$ $(\mathrm{p}<0,05),($ у городских с $1,3 \pm 0,4 \%$ в 1991 до $3,2 \pm 0,6 \% 0000$ в 2014 г. $(\mathrm{APC}=4,1 \quad(3,3-4,94) \%$ $(\mathrm{p}<0,05)$ и сельских с $1,2 \pm 0,55 \%$ в 1991 до $2,7 \pm 0,9^{0} \%$ о000 в 2014 г. $($ APC $=3,1 \quad(2,08-4,06) \%$ $(\mathrm{p}<0,05))$. При этом у женщин показатель заболеваемости вырос с $1,5 \pm 0,33 \%$ в $3,3 \pm 0,5 \%$ о000 в 2014 г. $($ APC $=3,9(3,24-4,65) \%$ $(\mathrm{p}<0,05),($ у городских с $1,4 \pm 0,39 \%$ $3,4 \pm 0,58 \%$ \%000 в 2014 г. $($ APC $=4,2(3,39-4,93) \%$ $(\mathrm{p}<0,05)$ и сельских с $1,7 \pm 0,59 \%$ в 1991 до $3,1 \pm 1,02 \%$ в 2014 г. $(\mathrm{APC}=3,4(2,17-4,67) \%$ $(\mathrm{p}<0,05))$. Заболеваемость ММ на протяжении всего периода наблюдения у женщин была незначительно выше, чем у мужчин. При этом следует отметить, что заболеваемость ММ у городских жителей мужчин и женщин была незначительно выше, чем у сельских. Статистически значимые различия $(\mathrm{p}<0,05)$ грубых интенсивных показателей заболеваемости ММ у мужчин и женщин отмечаются в 2001, 2005, 2007 и 2010 гг. В то же время статистически значимые различия $(\mathrm{p}<0,05)$ грубых интенсивных показателей заболеваемости ММ у мужчин, городских и сельских жителей отмечались в 1997-м и 2011 гг. и у женщин в 1998-м и 2001 гг.

Как видно из таблицы, динамика стандартизованного показателя имеет сходство с динамикой грубого интенсивного показателя. Статистически значимый рост показателей ASR отмечается у мужчин и женщин, как городских, так и сельских жителей. Стандартизованные показатели заболеваемости у мужчин вырос и составил $1,2 \pm 0,17 \%$ в000 в 1991 г. и $2,2 \pm 0,2 \%$ в 2014 г. (АРC $=3 \quad(2,3-3,68) \% \quad(p<0,05), \quad(y \quad$ городских -
$1,5 \pm 0,24 \%$ в000 в 1991 и $2,5 \pm 0,25 \%$ в 2000 в 2014 г. $(\mathrm{APC}=2,5 \quad(1,61-3,42) \%(\mathrm{p}<0,05)$ и сельских $1 \pm 0,34 \% 0000$ в 1991 и $1,6 \pm 0,37^{0} \% 000$ в 2014 г. $(\mathrm{APC}=2,8(1,59-4,03) \%(\mathrm{p}<0,05))$. При этом у женщин стандартизованный показатель заболеваемости вырос с $1 \pm 0,15 \%$ о $1,7 \pm 0,28 \%$ в 2000 в 2014 . $($ PPC $=2,8(2,09-3,55) \%$ $(\mathrm{p}<0,05)$, однако у городских и сельских жителей он менялся незначительно, составляя соответственно $1,2 \pm 0,21 \%$ в 1991 и $1,9 \pm 0,42 \%$ в 2014 г. $(\mathrm{APC}=2,3(1,58-3,01) \%$ $(\mathrm{p}<0,05)$ и $0,8 \pm 0,23 \%$ о 2014 г. $($ АРС $=3,1(1,75-4,48) \%(p<0,05))$. Следует отметить, что, как и в случае грубых интенсивных показателей заболеваемости, стандартизованные по возрасту показатели выше у городских, чем у сельских жителей, как у мужчин, так и у женщин. Однако стандартизованный показатель заболеваемости оказался незначительно выше у мужчин, чем у женщин, в отличие от грубых интенсивных показателей.

Статистически значимые различия $(\mathrm{p}<0,05)$ стандартизованных по возрасту показателей заболеваемости между мужчинами и женщинами отмечены в 1991-1994 и 1991-2014 гг. В то же время статистически значимые различия отмечались между мужчинами города и села и женщинами во все годы наблюдения, за исключением 2005-2009 гг. у мужчин и в 2000-2004 гг. у женщин городских и сельских жителях.

Таким образом, прроведенный анализ заболеваемости за период с 1991-го по 2014 год позволяет сделать выводы о росте заболеваемости ММ у населения Республики Беларусь в послеаварийный период. Среднегодовой темп прироста составляет порядка 3,5\%.

Анализ повозрастных показателей заболеваемости показал, что риск заболеть ММ в первые 30 лет жизни минимален. После 35 лет риск заболеть ММ резко увеличивается с возрастом, достигая максимума в возрастной группе 70-74 года у мужчин $(11 \pm 1,19 \%$ \%о00) и у женщин $(9,2 \pm 0,78 \%$ о0о0). После 75 лет заболеваемость ММ начинает снижаться.

Грубый интенсивный показатель заболеваемости ММ незначительно выше у женщин, чем у мужчин, и у жителей города, чем села.

Анализ стандартизованных по возрасту показателей показал, что в отличие от грубых интенсивных показателей заболеваемость ММ выше у мужчин, чем у женщин. Однако, как и у грубых интенсивных показателей заболеваемости, стандартизованные по возрасту показатели выше у городских, чем у сельских жителей, как у мужчин, так и у женщин. 


\section{ЛИТЕРАТУРА}

1. Биологические эффекты при облучении в малых дозах. Источники и эффекты ионизирующего излучения. Отчет НКАДР 2000 г. Генеральной Ассамблее ООН с научными приложениями. Т. 2 : Эффекты (Ч. 3) / Пер. с англ. - М. : РАДЭКОН, 2000. - С. 215.

2. Моисеев П.И., Веялкин И.В., Демидчик Ю.Е. Эпидемиология злокачественных новообразований: принципы и методы // Руководство по онко- логии: учебник / О.Г. Суконко [и др.]; под ред. О.Г. Суконко. - Минск, 2015. - С. 51-82.

3. Breslow N.E., Day N.E. Statistical methods in cancer research. Volume II -- The design and analysis of cohort studies. - Lyon: IARC, 1987. - Vol. 2. $404 \mathrm{p}$.

4. Globocan 2012: Estimated cancer incidence, mortality and prevalence worldwide in 2012 [Electronic resource] / Interntional Agency for Research of cancer. - France, 2012. - mode of access: http://globocan.iarc.fr. - date of access: 12.01.2016. 